\title{
T1 Mapping for Quantifying Myocardial Viability in the Peri-Infarct Regions
}

\author{
Yuko Tada and Phillip C Yang* \\ Cardiovascular medicine, Stanford University School of Medicine, USA
}

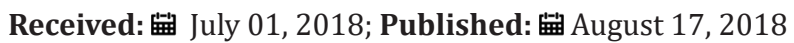

*Corresponding author: Phillip C Yang, Stanford University School of Medicine, 300 Pasteur Drive, Stanford CA94305, USA

\begin{abstract}
Abbreviations: DEMRI: Delayed Gadolinium Enhancement MRI; STEMI: ST Elevation Myocardial Infarction; AMI: Acute Myocardial Infarction; LV: Left Ventricular; IC: Infarct Core; PIR: Peri Infarct Region; IR: Ischemia Reperfusion Injury; MVO: Microvascular Obstruction; IMH: Intramyocardial Hemorrhage; T2WI: T2 Weighted MRI; ECV: Extra Cellular Volume; MEMRI: Manganese Enhanced MRI
\end{abstract}

\section{Introduction}

While the advances in revascularization therapy have drastically decreased the mortality following ST elevation myocardial infarction (STEMI), the number of patients who develops heart failure (HF) following the reperfusion therapy continues to rise. More than $30 \%$ of the patients who survive STEMI develop HF long term [1]. In addition, patients with a history of acute myocardial infarction (AMI) and left ventricular (LV) dysfunction have a markedly increased 6 months mortality of greater than 10\% [2] Thus, there is an urgent unmet clinical need to clarify the mechanisms of SCD and HF post-MI and develop a robust biomarker to identify the high-risk patients. The importance of differentiating the periinfarct region (PIR) from the adjacent non-viable infarct core (IC) has been noted recently as it could independently determine the occurrence of major adverse clinical events [3,4]. The PIR consisting of heterogeneous tissue components represents the nexus for active remodeling or repair characterized by extracellular matrix formation, angiogenesis, oxidative stress, mitochondrial energetics, apoptosis and inflammation $[5,6]$. We here review the non-invasive MRI approaches to characterize PIR.

\section{MRI Characterization of PIR}

Delayed gadolinium enhancement MRI (DEMRI) is the current gold standard to assess the non-viable myocardial infarct. However, up to $10 \%$ of the DEMRI positive myocardial infarct includes histologically viable PIR represented by reduced signal of the gadolinium based contrast agent [7-9]. The ischemia reperfusion injury (IR) results in the formation of heterogeneous tissues, containing vulnerable cardiomyocytes in the PIR9. However, the exact relationship between the IR injury and formation of PIR remains unknown. The pathological changes associated with IR injury such as myocardial edema, microvascular obstruction (MVO) and intramyocardial hemorrhage (IMH) delineated by DEMRI and
T2 weighted MRI (T2WI) correlate with the major adverse cardiac events (MACE) $[10,11]$. Their predictive capability of LV arrhythmia and SCD in the post MI period has not yet been determined. Recent advances in T1 mapping technique could help characterize the PIR more precisely. Pathological changes, including fibrosis, necrosis and edema increase pre-contrast (native) T1 value of the myocardium. It contains both myocytes and connective tissue information. Expansion of the extra cellular volume (ECV) fraction in the infarct injury region causes a greater change in the MRI relaxation (R1) value after the administration of the gadoliniumbased extracellular contrast agent when compared to the remote myocardium [12]. The ECV of the myocardium is calculated by measuring Hct and T1 for the blood pool: ECV = (1-Hct) $\mathrm{x}$ (R1postR1pre) myo/ (R1post-R1pre) blood, where R1 equals 1/T1 [13]. Diagnostic values of native T1 and ECV in the acute myocardial infarction in predicting $\mathrm{LV}$ dysfunction have been indicated in several clinical studies [14-16]. However, one of the intrinsic disadvantages is that the ECV does not detect the dysfunctional myocardium directly.

\section{The Approach to PIR Using Manganese Enhancement MRI and T1 Mapping}

An alternative approach, manganese enhanced MRI (MEMRI), uses manganese $(\mathrm{Mn} 2+$ ) based biological contrast agent to represent the viability of cardiomyocytes directly through the ability of individual cells to take up manganese ions through L-type calcium channels [17]. MEMRI distinguishes the injured but viable myocardium in the infarct tissue to assess the extent of salvageable myocardium in the heterogeneous PIR. We previously showed that the PIR can be delineated by the dual MEMRI and DEMRI contrast where the MEMRI positive viable area is located inside the DEMRI positive myocardial infarct $[18,19]$. DEMRI positive total infarct can be divided into non-viable MEMRI negative infarct core (IC) 
and viable MEMRI positive PIR territories (Figure 1). In addition, T1 mapping of the native and MEMRI-enhanced myocardium could delineate and quantitate the viability of PIR. Furthermore, the rapid first pass uptake of manganese by the cardiomyocytes measures the R1 change (delta R1) before and after MEMRI to represent the specific viability of the cardiomyocytes [20,21]. Thus, myocardial injury in the heterogeneous infarct zones can be assessed based on the differential myocardial cellular viability using T1 mapping before and after MEMRI [22].

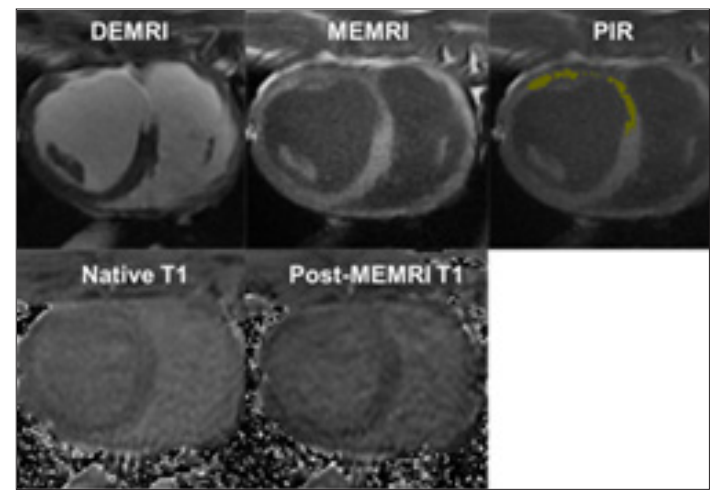

Figure 1: PIR characterization using DEMRI, MEMRI and T1 mapping.

Note: The LAD IR injury model was created in a swine and MRI was performed 4 weeks later. DEMRI positive total infarct was divided into non-viable MEMRI negative infarct core (IC) and viable MEMRI positive PIR (yellow). T1 mapping was performed using smarT1map sequence (GE Healthcare) before and after MEMRI. Native T1 and post-MEMRI T1 of each infarct territory can be calculated.

\section{Preclinical and Clinical Applications}

The viability signal may indicate LV remodeling and arrhythmia potential more strongly compared to the assessment of necrosis or fibrosis by native T1 or ECV mapping. Clinical trials in ischemic cardiomyopathy patients are on-going in our institution. Our preclinical study using swine IR injury model characterized the heterogeneous infarct tissues consisting of the infarct core (IC) and PIR based on native T1 value and myocardial viability. The analysis revealed IC, PIR, and remote zone all had distinct native T1, post MEMRI T1, and delta R1 values. Utilizing these novel contrast techniques, roles of IR injury in the formation of inflammatory, heterogeneous PIR, resulting in eventual MACE will be elucidated and a reliable non-invasive imaging modality to predict the occurrence of ventricular arrhythmia will be developed.

\section{Conclusion}

Characterization of myocardial viability in the PIR is feasible using T1 mapping post MEMRI. Unique strengths of each contrast agent must be leveraged to better understand the role of the PIR in $\mathrm{HF}$ and SCD.

\section{References}

1. Bui AL, Horwich TB, Fonarow GC (2011) Epidemiology and risk profile of heart failure. Nat Rev Cardiol 8(1): 30-41.

2. Burns RJ, Gibbons RJ, Yi Q Roberts RS, Miller TD, et al. (2002) The relationships of left ventricular ejection fraction, end-systolic volume index and infarct size to six-month mortality after hospital discharge following myocardial infarction treated by thrombolysis. J Am Coll Cardiol 39(1): 30-36.

3. Yokota H, Heidary S, Katikireddy CK, Nguyen P, Pauly JM, et al. (2008) Quantitative characterization of myocardial infarction by cardiovascular magnetic resonance predicts future cardiovascular events in patients with ischemic cardiomyopathy. J Cardiovasc Magn Reson 10:17.

4. Heidary S, Patel H, Chung J, Yokota H, Gupta SN, et al. (2010) Quantitative tissue characterization of infarct core and border zone in patients with ischemic cardiomyopathy by magnetic resonance is associated with future cardiovascular events. J Am Coll Cardiol 55(24): 2762-2768.

5. Yang L, Gregorich ZR, Cai W, Zhang P, Young B, Gu Y, Zhang J, et al. (2017) Quantitative Proteomics and Immunohistochemistry Reveal Insights into Cellular and Molecular Processes in the Infarct Border Zone One Month after Myocardial Infarction. J Proteome Res 16(5): 2101-2112.

6. Duran JM, Taghavi S, Berretta RM, Makarewich CA, Sharp Iii T, et al. (2012) A characterization and targeting of the infarct border zone in a swine model of myocardial infarction. Clin Transl Sci 5(5): 416-421.

7. Saeed M, Bremerich J, Wendland MF, Wyttenbach R, Weinmann HJ, et al. (1999) Repeused myocardial infarction as seen with use of necrosisspecific versus standard extracellular MR contrast media in rats. Radiology 213: 247-257.

8. Saeed M, Lund G, Wendland MF, Bremerich J, Weinmann H, et al. (2001) Magnetic resonance characterization of the peri-infarction zone of reperfused myocardial infarction with necrosis-specific and extracellular nonspecific contrast media. Circulation 103(6): 871-876.

9. Roifman I, Ghugre NR, Vira T, Zia MI, Zavodni A, et al. (2016) Assessment of the longitudinal changes in infarct heterogeneity post myocardial infarction. BMC Cardiovasc Disord 16(1): 198.

10. Robbers LF, Eerenberg ES, Teunissen PF, Jansen MF, Hollander MR, et al. (2013) Magnetic resonance imaging-defined areas of microvascular obstruction after acute myocardial infarction represent microvascular destruction and haemorrhage. Eur Heart J 34(30): 2346-2353.

11. Reichek N (2014) Meta-analysis of MACE in MI: what's the MO? JACC Cardiovasc Imaging 7(9): 953-955.

12. Ugander M, Oki AJ, Hsu LY, Kellman P, Greiser A, et al. (2012) Extracellular volume imaging by magnetic resonance imaging provides insights into overt and sub-clinical myocardial pathology. Eur Heart J 33(10): 12681278.

13. Flett AS, Hayward MP, Ashworth MT, Hansen MS, Taylor AM, et al. (2010) Equilibrium contrast cardiovascular magnetic resonance for the measurement of diffuse myocardial fibrosis: preliminary validation in humans. Circulation 122(2): 138-144.

14. Carberry J, Carrick D, Haig C, Rauhalammi SM, Ahmed N, et al. (2016) Remote Zone Extracellular Volume and Left Ventricular Remodeling in Survivors of ST-Elevation Myocardial Infarction. Hypertension. 68: 385691.

15. Kidambi A, Motwani M, Uddin A, Ripley DP, Mc Diarmid AK, et al. (2016) Myocardial Extracellular Volume Estimation by CMR Predicts Functional Recovery Following Acute MI. JACC Cardiovasc Imaging 10(9): 989-999.

16. Liu D, Borlotti A, Viliani D, Jerosch Herold M, Alkhalil M, et al. (2017) CMR Native T1 Mapping Allows Differentiation of Reversible Versus Irreversible Myocardial Damage in ST-Segment-Elevation Myocardial Infarction: An Ox AMI Study (Oxford Acute Myocardial Infarction). Circ Cardiovasc Imaging 10(8).

17. Krombach GA, Saeed M, Higgins CB, Novikov V, Wendland MF (2004) Contrast-enhanced MR delineation of stunned myocardium with administration of $\mathrm{MnCl}_{2}$ in rats. Radiology 230(1): 183-190.

18. Dash R, Chung J, Ikeno F, Hahn Windgassen A, Matsuura Y, et al. (2011) Dual manganese-enhanced and delayed gadolinium-enhanced MRI detects myocardial border zone injury in a pig ischemia-reperfusion model. Circ Cardiovasc Imaging 495: 574-482. 
19. Dash R, Kim PJ, Matsuura Y, Ikeno F, Metzler S, et al. (2015) ManganeseEnhanced Magnetic Resonance Imaging Enables In Vivo Confirmation of Peri-Infarct Restoration Following Stem Cell Therapy in a Porcine Ischemia-Reperfusion Model. J Am Heart Assoc 4(7)

20. Storey P, Danias PG, Post M, Li W, Seoane PR, et al. (2003) Preliminary evaluation of EVP 1001-1: a new cardiac-specific magnetic resonance contrast agent with kinetics suitable for steady-state imaging of the ischemic heart. Invest Radiol 38(10): 642-52.

ISSN: 2574-1241

DOI: $10.26717 / B J S T R .2018 .08 .001608$

Phillip C Yang. Biomed J Sci \& Tech Res

CC (P) This work is licensed under Creative

Submission Link: https://biomedres.us/submit-manuscript.php
21. Eriksson R, Johansson L, Bjerner T and Ahlström H (2005) Dobutamineinduced stress affects intracellular uptake of manganese: a quantitative magnetic resonance imaging study in pigs. J Magn Reson Imaging 21(4): 360-364.

22. Tada Y, Heidary S, Tachibana A, Sano H, Neofytou E, et al. (2017) Cardiac viability in the peri-infarct region quantified by T1 mapping following manganese-enhanced MRI (MEMRI) is associated with LV remodeling post-myocardial infarction (MI) SCMR.

\begin{tabular}{ll} 
BIOMEDICAL & Assets of Publishing with us \\
RESEARCHES & - Global archiving of articles \\
\hline Immediate, unrestricted online access \\
\end{tabular}

